\title{
Strain-modified RKKY interaction in carbon nanotubes
}

\author{
Gorman, P. D.; Duffy, J. M.; Power, Stephen R.; Ferreira, M.S.
}

Published in:

Physical Review B Condensed Matter

Link to article, DOI:

10.1103/PhysRevB.92.035411

Publication date:

2015

Document Version

Publisher's PDF, also known as Version of record

Link back to DTU Orbit

Citation (APA):

Gorman, P. D., Duffy, J. M., Power, S. R., \& Ferreira, M. S. (2015). Strain-modified RKKY interaction in carbon nanotubes. Physical Review B Condensed Matter, 92, [035411]. https://doi.org/10.1103/PhysRevB.92.035411

\section{General rights}

Copyright and moral rights for the publications made accessible in the public portal are retained by the authors and/or other copyright owners and it is a condition of accessing publications that users recognise and abide by the legal requirements associated with these rights.

- Users may download and print one copy of any publication from the public portal for the purpose of private study or research.

- You may not further distribute the material or use it for any profit-making activity or commercial gain

- You may freely distribute the URL identifying the publication in the public portal

If you believe that this document breaches copyright please contact us providing details, and we will remove access to the work immediately and investigate your claim 


\title{
Strain-modified RKKY interaction in carbon nanotubes
}

\author{
P. D. Gorman, ${ }^{1, *}$ J. M. Duffy, ${ }^{1}$ S. R. Power, ${ }^{2}$ and M. S. Ferreira ${ }^{1,3}$ \\ ${ }^{1}$ School of Physics, Trinity College Dublin, Dublin 2, Ireland \\ ${ }^{2}$ Center for Nanostructured Graphene (CNG), DTU Nanotech, Department of Micro- and Nanotechnology, \\ Technical University of Denmark, DK-2800 Kongens Lyngby, Denmark \\ ${ }^{3}$ CRANN, Trinity College Dublin, Dublin 2, Ireland \\ (Received 5 May 2015; published 9 July 2015)
}

\begin{abstract}
For low-dimensional metallic structures, such as nanotubes, the exchange coupling between localized magnetic dopants is predicted to decay slowly with separation. The long-range character of this interaction plays a significant role in determining the magnetic order of the system. It has previously been shown that the interaction range depends on the conformation of the magnetic dopants in both graphene and nanotubes. Here we examine the Ruderman-Kittel-Kasuya-Yosida (RKKY) interaction in carbon nanotubes in the presence of uniaxial strain for a range of different impurity configurations. We show that strain is capable of amplifying or attenuating the RKKY interaction, significantly increasing certain interaction ranges, and acting as a switch: effectively turning on or off the interaction. We argue that uniaxial strain can be employed to significantly manipulate magnetic interactions in carbon nanotubes, allowing an interplay between mechanical and magnetic properties in future spintronic devices. We also examine the dimensional relationship between graphene and nanotubes with regards to the decay rate of the RKKY interaction.
\end{abstract}

DOI: 10.1103/PhysRevB.92.035411

PACS number(s): 73.63.Fg, 75.30.Hx, 62.20.-x, 75.75.-c

\section{INTRODUCTION}

Recently, there has been much investment in the field of spintronics motivated by the tremendous potential for technological applications. Low-dimensional structures such as graphene [1], nanowires [2], nanotubes [3], nanoribbons [4], silicene [5], and many more are expected to lead to useful spintronic applications, possibly leading to the production of extremely efficient magnetic sensors, high-capacity memory storage, and nonvolatile computer memories [6,7]. Important to spintronics is the mechanism of interaction between embedded impurities known as the indirect exchange interaction.

This interaction is one of many such interactions mediated by the conduction electrons of the host material and is realized as the energetically favorable configurations of localized moments, driven by the energy difference between them. Usually calculated within the Ruderman-Kittel-KasuyaYosida (RKKY) approximation [8-13], this interaction has been extensively studied in graphene [14-31] and carbon nanotubes [32-36], where the focus has been on the sign, magnitude, and rate of decay of the interaction [14,37-42]. This type of analysis has been carried out for the 4 main types of impurities: substitutional, top adsorbed, bridge adsorbed, and center adsorbed (Fig. 1). These impurities are differentiated by their conformation with the lattice, which is found to have a strong effect on the behavior of the RKKY interaction [23,24,27,28,39,41]. In carbon nanotubes (CNTs) the RKKY interaction is thought to decay as $D^{-1}$, where $D$ is the separation between impurities. This is the case for substitutional, top-, and bridge-adsorbed impurities, implying that the magnetic moments of adatoms are able to feel their mutual presence even when they are very far apart. For centeradsorbed impurities (also known as plaquette impurities) the

*pgorman@tcd.ie interaction is predicted to have a $D^{-5}$ decay rate [33]. This decay rate is significantly faster than for other impurity types, and is not as useful for applications. This is unfortunate as it is the preferred configuration for many common transition metals, which are likely magnetic dopants [43-46].

Controlling the interaction between magnetic objects in carbon nanotubes (CNTs) may provide an entry into the technologically promising area of spintronics. In order to expand the applicability of those systems we need to understand how the interactions may be modified to suit our needs. Much progress has been made explaining the intrinsic properties of nanotubes and nearly two decades after their discovery CNTs are still the subject of intensive scientific research due to their intriguing physical properties [47-49]. One avenue that has been explored to alter the intrinsic properties of nanotubes is the introduction of strain. The effects of strain on the elastic, structural, and electronic properties of carbon nanotubesincluding band gap and electron conductance-have been extensively studied [50-56]. Many uses have been suggested for strained nanotubes including strain sensors and field-effect transistors $[57,58]$. It has been shown that uniaxial strain can open a band gap in metallic nanotubes and alter the band gap in semiconducting nanotubes $[59,60]$. One avenue that has not been explored is how strain may affect their magnetic properties.

It has been shown that the properties of RKKY interaction in both graphene $[24,38,40]$ and nanotubes $[32,33]$ are strongly influenced by the conformation of the magnetic impurities with the lattice. In graphene it has been shown that uniaxial strain has the ability to amplify or attenuate that interaction, as well massively increase the range or suppress the interaction in some cases $[38,40,61,62]$. In graphene nanoribbons strain has been shown to tune the exchange splitting of nonvanishing moments induced by vacancies in the lattice [63]. In this paper we determine the role that strain plays in controlling the RKKY interaction in achiral carbon nanotubes, and explore 
(a)

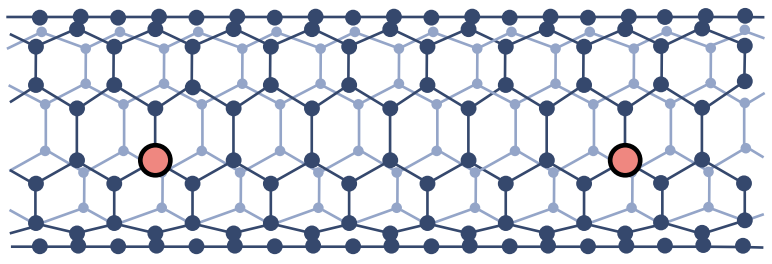

(b)

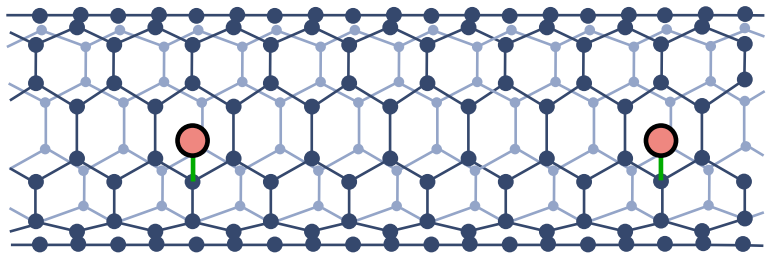

(c)

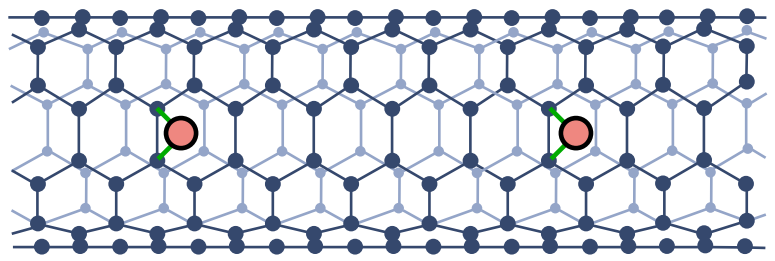

(d)

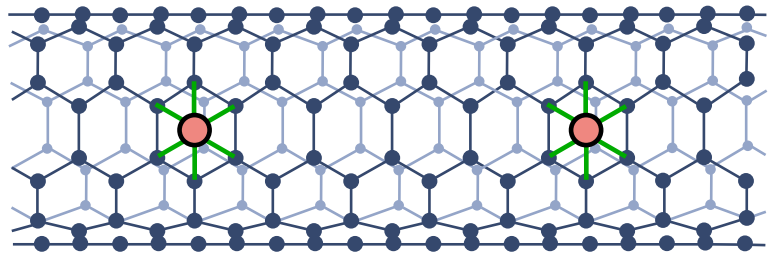

(e)

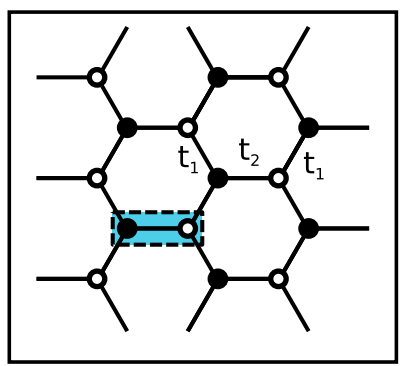

FIG. 1. (Color online) A schematic of an armchair nanotube with impurities, represented by the red circle, whose interactions with the nanotube lattice are indicated by the solid green lines. Four different types of impurities are represented: (a) substitutional impurities, (b) top-adsorbed impurities, (c) bridge-adsorbed impurities, and (d) center-adsorbed impurities. (e) A schematic of the unit cell, highlighted in blue, and the interatomic hopping integrals $t_{2}$ and $t_{1}$.

the possibility of using strain to modify the interaction between such impurities. We examine the four main types of impurity conformations: substitutional, where a carbon atom is replaced by an impurity; top adsorbed, where an impurity sits above, and connects to, a single carbon atom; bridge adsorbed, where an impurity sits above the bond between two neighboring carbon atoms; and center adsorbed, where an impurity sits at the center of a carbon hexagon and attaches equally to all six surrounding carbons. This work is carried out for both armchair-edged nanotubes (ACNTs) and achiral zigzag-edged nanotubes (ZZNTs) as they have substantially different reactions to strain. Figure 1 schematically shows the four conformation types in an achiral ACNT, where the impurities are separated along the axial direction of the tube. We provide simple expressions for the interactions between two impurities on a strained nanotube. The simplicity of these expressions allows us to understand the modified coupling in terms of the intercarbon hopping integrals, and analytically understand how strain may change the decay rate drastically. We also explore the role played by nanotube circumference in determining the decay rate of the RKKY interaction between impurities in order to validate our model.

\section{METHODS}

The energy difference between the ferromagnetic (FM) and antiferromagnetic (AFM) alignments of two moments embedded in a conducting host is described as the indirect exchange coupling between the two moments. The Lloyd formula method allows us to calculate the total energy difference, $J_{A B}$, between two magnetic impurities labeled $A$ and $B$. The Lloyd formula is given by

$$
J_{A B}=-\frac{1}{\pi} \operatorname{Im} \int d E f(E) \ln \left[1+4 V_{e x}^{2} g_{a b}^{\sigma}(E) g_{b a}^{\sigma}(E)\right],
$$

where $f(E)$ is the Fermi function, $V_{e x}$ is a spin-dependent on-site potential that accounts for the exchange splitting in the magnetic orbitals, and $g_{a b}^{\sigma}(E)$ is the real-space, single-electron Green's function (GF) describing the propagation of electrons with spin $\sigma=\uparrow$ or $\downarrow$. According to our definition $J<0$ $(J>0)$ corresponds to a ferromagnetic (antiferromagnetic) alignment of magnetic moments. Our nanotube is modeled using the nearest-neighbor tight-binding approximation with an interatomic hopping integral $t_{0}=-2.7 \mathrm{eV}$, which provides a good approximation of the electronic structure of CNTs for all but the smallest circumference tubes. Unless otherwise specified we will use $t_{0}$ as our energy unit.

We assume that each substitutional impurity causes a change in on-site energy, and that each adsorbed impurity orbital has a finite hopping $\tau$ to $N$ adjacent sites on the graphene lattice: top adsorbed $(N=1)$, bridge adsorbed $(N=2)$, and center adsorbed $(N=6)$. We will only consider a single magnetic orbital at each impurity site, separated along the longitudinal direction of the nanotube; however, it is straightforward to generalize this approach to deal with multiple orbitals, or separations with an axial component. We will not deal with the exact parametrizations for specific impurity types here. These parametrizations can be found in numerous $a b$ initio studies $[43,45,46]$ of single impurities in nanotubes with different configurations, though it is worth noting that the results presented here are not strongly dependent on the impurity type used.

Strain, $\varepsilon$, is introduced into the nanotube system in a similar manner as for a graphene system [38,40,64,65], by splitting the hopping integral between carbon atoms, $t_{0}$, into straindependent hopping integrals $t_{2}(\varepsilon)$ and $t_{1}(\varepsilon)$. In the convention used here $t_{2}$ is the intra-unit-cell hopping and $t_{1}$ is the inter-unitcell hopping [Fig. 1(e)], and the dependency on the strain is usually omitted for neatness. The strain-dependent, real-space GF between two sites on the graphene lattice can be written as 
a double integral over the Brillouin zone [26,32] in the form

$$
g_{a b}^{\sigma}=\frac{1}{2 \pi^{2}} \int_{-\pi / 2}^{\pi / 2} d k_{Z} \int_{-\pi}^{\pi} d k_{A} \frac{N_{\gamma}(E, \mathbf{k}) e^{i \mathbf{k} \cdot \mathbf{D}}}{E^{2}-|f(\mathbf{k})|^{2}},
$$

where

$$
f(\mathbf{k})=t_{2}+2 t_{1} \cos \left(k_{Z}\right) e^{i k_{A}} .
$$

Here $N_{\gamma}$ contains information about the sites of each impurity, $D$ is a separation vector, and $f(\mathbf{k})$ is a sum of Bloch phase terms over nearest neighbors.

The Green's function (GF) for nanotubes is derived in a similar manner to that of the GF for pristine graphene, except that the periodicity in the nanotube causes one of the components of one of the $k$ vectors to be quantized. The integral then becomes a sum over all the unique $k$ points, which are obtained from the quantization condition which describes the circumference of the nanotube, $n_{c}$. Following the procedure of Ref. [32] the GFs between two sites for both ACNTs and ZZNTs may now be written in a general form

$$
\mathcal{G}_{a b}=\sum_{j} \mathcal{A}(E, j) e^{i \mathcal{Q}(E, j) D} .
$$

We denote the GF as $\mathcal{G}_{a b}$ for nanotubes to distinguish it from the GF for graphene, which we denoted $g_{a b}$. This will now allow us to solve the Lloyd formula analytically.

To calculate the coupling between two impurities, $A$ and $B$, we now use the RKKY approximation to write Eq. (1) as

$$
J \sim \operatorname{Im} \int d E f(E) V_{e x}^{2} \mathcal{G}_{A B}^{2} .
$$

Using the general form of our GFs we can simplify our coupling to

$$
J \sim \sum_{j, k} \operatorname{Im} \int d E f(E) V_{e x}^{2} \mathcal{B}(E, j, k) e^{i 2 \mathcal{Q}(E, j, k) D},
$$

where $\mathcal{B}(E, j, k)=\mathcal{A}(E, j) \mathcal{A}(E, k)$.

We have shown previously that the magnetic coupling can be easily extracted when the coupling is expressed in such a form, by reducing the integration to a sum over Matsubara frequencies and expanding the functions $\mathcal{B}(E)$ and $\mathcal{Q}(E)$ around the Fermi energy in the low-temperature limit $[38,40]$. Note that $\mathcal{B}(E)$ and $\mathcal{Q}(E)$ are also strain-dependent, but the variable $\varepsilon$ has been omitted for neatness. The coupling between impurities can then be expressed as

$$
J \sim-V_{e x}^{2} \sum_{j, k} \operatorname{Im}\left(e^{2 i \mathcal{Q}^{(0)} D} \sum_{l}\left[\frac{\mathcal{B}^{(l)}}{\left(2 i \mathcal{Q}^{(1)}\right)^{l+1}} \frac{1}{D^{(l+1)}}\right]\right),
$$

where the dependence of $\mathcal{Q}$ and $\mathcal{B}$ on $E, j, k$ has been omitted for simplicity.

\section{RKKY INTERACTION AS A FUNCTION OF NANOTUBE CIRCUMFERENCE}

It has previously been reported that in nanotubes the RKKY interaction between substitutional, top-adsorbed, and bridgeadsorbed impurities decays as $D^{-\alpha}$ with $\alpha=1$, and $\alpha=5$ for center-adsorbed impurities, where $D$ is the separation between

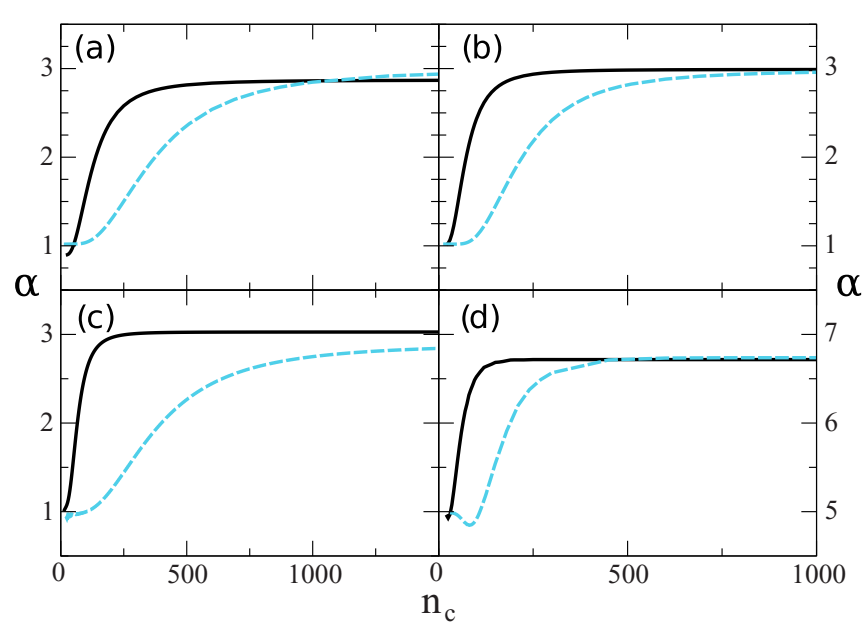

FIG. 2. (Color online) Decay $(\alpha)$ versus circumference $\left(n_{c}\right)$ for ACNT (solid black) and ZZNT (dashed blue) for (a) substitutional impurities, same sublattice; (b) substitutional impurities, opposite sublattice; (c) bridge adsorbed; (d) center adsorbed.

the impurities [33]. However these have been calculated without much regard for the effect that the circumference of the nanotube has on the interaction. In bulk graphene these rates are found to be $\alpha=3$ for substitutional, top adsorbed, and bridge adsorbed, and $\alpha=7$ for center adsorbed [40]. In this section we examine the role of the nanotube circumference, $n_{c}$ (which can be easily related to the more usual measure of diameter), for both armchair nanotubes and metallic zigzag nanotubes, to understand how the RKKY interaction transitions from the behavior predicted in nanotubes to that predicted in bulk graphene. Here $n_{c}$ is a dimensionless quantity which describes the number of vectors between unit cells, in the armchair (zigzag) direction for ACNTs (ZZNTs), that are required to traverse the circumference of the nanotube.

Figure 2 shows the change in decay, $\alpha$, between impurities as a function of the circumference for both ACNT and ZZNT at $E_{F}=0$. For both same- and opposite-lattice substitutional impurities and bridge-adsorbed impurities we see that the greatest predictor of behavior is whether the nanotube is an ACNT or a ZZNT. On ACNTs the interaction very quickly decays away from the nanotube case of $\alpha=1$ to the graphene case of $\alpha=3$. This is seen in panels (a), (b), and (c) as the black line, which resembles graphene by about $n_{c}=200$. On ZZNTs the interaction has a plateau of NT behavior before moving gradually to the behavior seen in graphene; this is shown as the blue line, and does not resemble graphene until about $n_{c}=1000$.

Figure 2(d) shows the same effect for center-adsorbed impurities. By increasing the circumference of the NT we show that both ACNT and ZZNT decay rates go from $D^{-5}$ to $D^{-7}$. Again, impurities on ACNT decay much more rapidly towards the bulk case and on ZZNT exhibit a plateau and a slow decay towards bulk. In ACNTs the axial separation is along the zigzag direction, which is known to feature a repeating triplet pattern of interaction strength: strong, strong, weak [32]. The circumference was increased in steps of three to produce a smooth curve. This result shows a geometry dependence in the transition from nanotube-like to graphene-like behavior as 


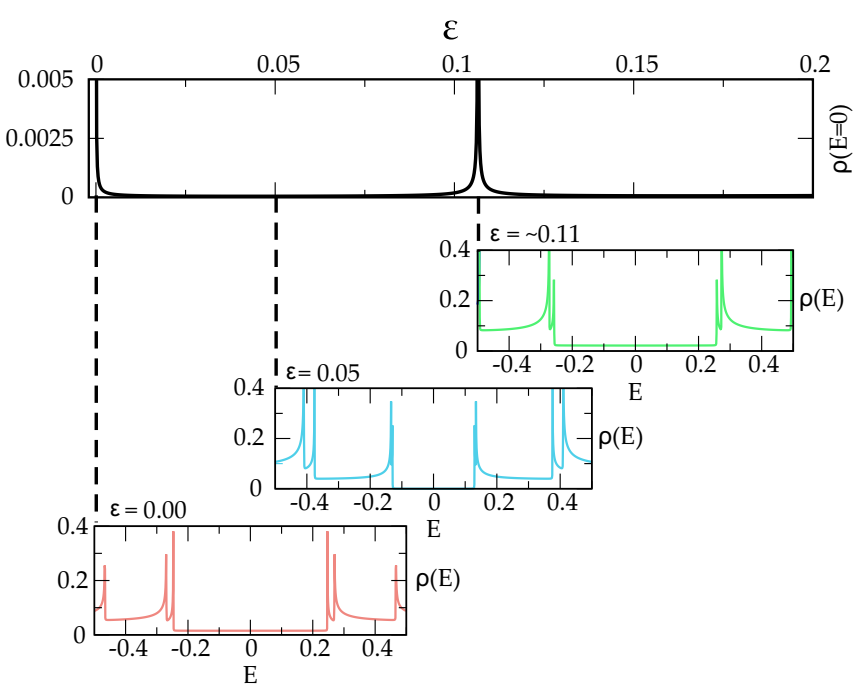

FIG. 3. (Color online) The density of states, $\rho$, at $E=0$ for a ZZNT with $n_{c}=21$ under uniaxial strain, $\varepsilon$. Subfigures show the density of states against energy for three strain values: $\varepsilon=0$ (red), $\varepsilon=0.05$ (blue), and $\varepsilon \approx 0.11$ (green).

the diameter of the CNT increases, and may be relevant for studies of the largest feasible CNTs since there is a notable difference between the two geometries even at quite small $n_{c}$.

\section{AXIAL STRAIN EFFECTS ON RKKY INTERACTION IN NANOTUBES}

Unstrained achiral nanotubes may be metallic or semiconducting. ACNTs are known to be metallic for all circumferences, $n_{c}$, whereas ZZNTs are only metallic if $n_{c}=3 k$, where $k$ is an integer, and are otherwise semiconducting. These properties are due to the intersection of the discretized momentum values and the Fermi surface. The introduction of strain, $\varepsilon$, into the nanotube system alters the coincidence of the momentum values and the Fermi surface; this in turn changes the electronic properties of nanotubes. ACNTs remain metallic under strain; however strain can change a conducting ZZNT into an insulating one, and conversely an insulating one into a conducting one [59]. Figure 3 shows the density of states (DOS) of a ZZNT with $n_{c}=21$ as strain is applied. The initially metallic NT immediately becomes semiconducting with slight strain, and as strain is further applied the two peaks in density of states come together and eventually merge. This is seen in all circumferences of semiconducting ZZNTs, where the precise value of strain required to shift the density of states away from zero around $E_{F}=0$ decreases as the circumference increases.

\section{A. Armchair-edged nanotubes}

Although ACNTs remain metallic with strain, this does not mean that the RKKY interaction is unchanged. Figure 4 shows the effect of strain on the RKKY interaction for substitutional and bridge-adsorbed impurities for several separations $D$, on an ACNT with $n_{c}=21$. For substitutional impurities it is noted that the magnitude of the opposite-sublattice interaction

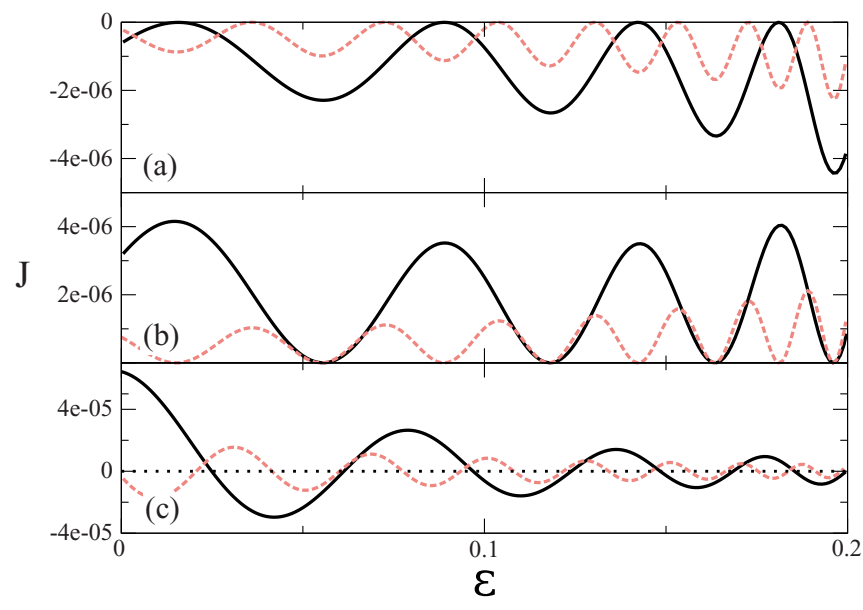

FIG. 4. (Color online) Plot of the coupling $(J)$ as a function of strain $(\varepsilon)$ for ACNTs at $D=10$ (black) and $D=20$ (dashed red) for (a) same-sublattice substitutional, (b) opposite-sublattice substitutional, and (c) bridge-adsorbed impurities, where the dotted black line indicates the transition from FM to AFM.

is larger than the same-sublattice interaction in the absence of strain. Top-adsorbed impurities have been omitted due to the similarity of behavior with substitutional impurities, including these sublattice effects. In all three cases oscillations are seen, but only in (c) are these oscillations around zero signifying a change from ferromagnetic to antiferromagnetic behavior. For both (a) same-sublattice and (b) oppositesublattice cases, strain is seen to either amplify or attenuate the interaction depending on the precise value of strain. This allows for the magnitude of these interactions to be precisely controlled. Interestingly there exist strain values (well within the experimental limits) that completely shut off the opposite-sublattice interaction, while simultaneously maximizing the same-sublattice interaction. In addition to this strain seems to generally amplify the interaction, while leaving the decay rate unchanged. This is understood from Eq. (7). The overall decay rate can be found from the first nonvanishing $\mathcal{B}(\varepsilon)$, which, for same-sublattice substitutional impurities, is

$$
\mathcal{B}(\varepsilon)^{(0)}=\frac{1}{4 n_{c}^{2}\left(t_{2}^{2}-4 t_{1}^{2}\right)}
$$

This factor corresponds to a coupling decay rate of $\alpha=1$, identical to that of the unstrained case. The equivalent opposite-sublattice term is similar. Small values of strain will not cause this term to vanish, and so no change in the decay rate is expected.

For bridge-adsorbed impurities [Fig. 4(c)] strain is capable of switching the sign of an interaction in a very controlled fashion. Since bridge-adsorbed impurities display a coupling with alternating sign as a function of separation strain cannot create wholly FM or AFM interactions between multiple impurities. However, for a pair of impurities it is capable of switching the sign of the interaction. The coupling between bridge-adsorbed impurities is weakened with strain; however the decay rate is not affected. The first nonvanishing $\mathcal{B}(\varepsilon)$ has the same form as Eq. (8), differing only by a factor of 16 , and 


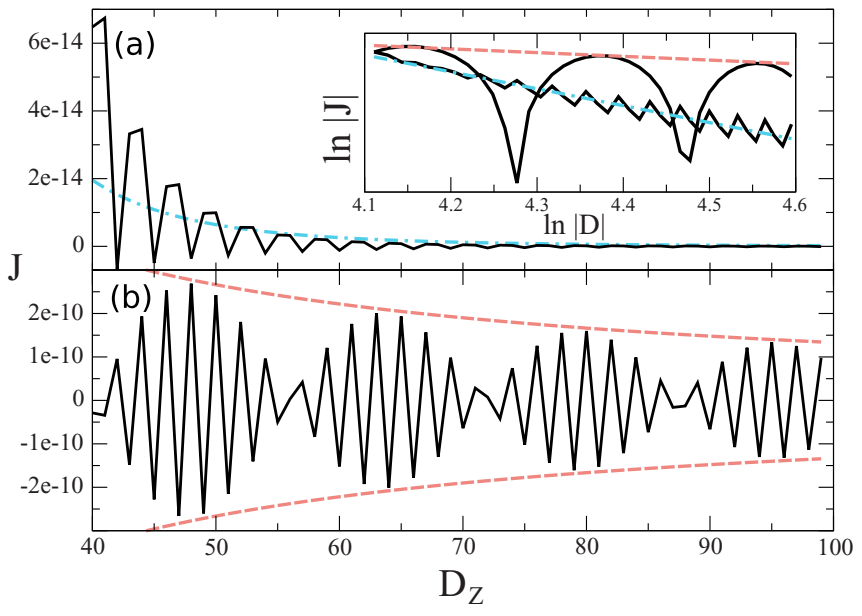

FIG. 5. (Color online) The interaction between two centeradsorbed impurities in an ACNT in (a) the absence of strain $(\varepsilon=0)$, and (b) the presence of strain $(\varepsilon=0.1)$. The inset is a normalized $\log$-log plot of both interactions. The colored lines show the decay rates: $D^{-5}$ (dash-dotted blue), $D^{-1}$ (dashed red).

as such it too corresponds to a decay rate of $D^{-1}$ for small strains.

For center-adsorbed impurities we see that the introduction of strain has a large impact on the decay rate and hence the magnitude of the interaction. Figure 5 shows the behavior of the interaction between center-adsorbed impurities with and without strain. Strain changes the interaction from being primarily antiferromagnetic to being split evenly between ferromagnetic and antiferromagnetic. The interaction between these impurities is known to decay rather quickly as $D^{-5}$ [Fig. 5(a)]. This can be understood from the $\mathcal{B}(\varepsilon)^{(l)}$ terms, which are zero for $l=0,1,2,3$. The first nonzero term is found at $\mathcal{B}(\varepsilon)^{(4)}$ and thus indicates the known decay rate of $D^{-5}$. The small values here owe to the necessity of examining the coupling at large ranges to ascertain a decay rate.

However, the introduction of strain breaks a key symmetry of the system massively increasing the range of the interaction. The interaction in the presence of strain decays slowly as $D^{-1}$ [Fig. 5(b)]. The inset of Fig. 5 shows a log-log plot of the coupling between the impurities for the unstrained and strained cases. Examining the $\mathcal{B}(\varepsilon)^{(0)}$ we find an explanation for the increased range,

$$
\mathcal{B}(\varepsilon)^{(0)}=\frac{\left(t_{2}-t_{1}\right)^{4} \phi(\varepsilon)}{4 n_{c}^{2} t_{1}^{4}\left(t_{2}^{2}-4 t_{1}^{2}\right)},
$$

where $\phi(\varepsilon)$ is a strain-dependent phase term. This simple expression allows us to understand the role that strain plays in the decay rate of center-adsorbed impurities. Here the numerator displays a $t_{2}-t_{1}$ term, since the unstrained system is defined by $t_{2}=t_{1}$ it is exactly zero only in unstrained graphene. Such a nonzero $\mathcal{B}(\varepsilon)^{(0)}$ term corresponds to a decay rate of $D^{-1}$. This symmetry-breaking effect is analogous to that seen in graphene where the decay rate is massively reduced from $D^{-7}$ to $D^{-3}$ by the introduction of strain [40]. This effect

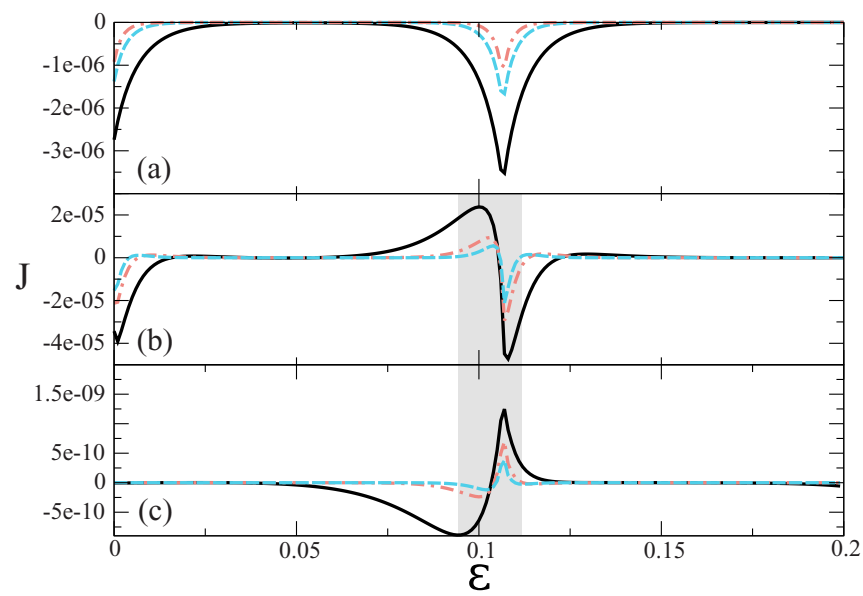

FIG. 6. (Color online) The coupling between (a) substitutional, (b) bridge-adsorbed, and (c) center-adsorbed impurities is plotted as a function of strain in a ZZNT with $n_{c}=21$. Each coupling is plotted for three values of separation $D=10$ (solid black), $D=20$ (dashed red), $D=30$ (dash-dotted blue). The gray bands indicate the regions of sudden FM/AFM switch.

allows the strength of the interaction to be massively increased with a relatively small amount of strain.

\section{B. Zigzag-edged nanotubes}

We now move our attention to ZZNTs. Since the introduction of strain changes a conducting ZZNT to a semiconducting ZZNT the effect of strain in ZZNTs is markedly different. Figure 6 shows the coupling between substitutional, bridgeadsorbed, and center-adsorbed impurities in ZZNT with $n_{c}=$ 21 as a function of strain. Since the introduction of strain changes a ZZNT from conducting to semiconducting the RKKY interaction in an initially conducting ZZNT goes to zero [Fig. 6(a)]. Strain also has the opposite effect on initially semiconducting ZZNTs, taking the RKKY away from zero for precise values of strain. Similar behavior is seen for bridge-adsorbed impurities [Fig. 6(b)], with the key difference being the sign change that occurs around this precise strain value. What this means is that for two bridge-adsorbed impurities separated by some distance $D$ there exists a small range of strain that can quickly turn the RKKY interaction from strongly AFM to strongly FM. This is in contrast to ACNTs where a sign change can be achieved but only gradually. The precise strain value will depend on the circumference of the nanotube $n_{c}$. In addition, the sign change occurs at all separations, meaning that it is possible for strain to quickly change a system of $N$ impurities from all having the same alignment to having alternating alignments leading to no overall magnetic moment. The center-absorbed impurity [Fig. 6(c)] shows similar behavior; however since a key symmetry that leads to the $D^{-5}$ decay is broken by strain the magnitude is increased massively, which gives the appearance of initially being zero. This could prove extremely useful for spintronic applications where it allows minor changes in strain to result in a large change in the magnetic ordering of impurities. This change could be implemented in a reversible and controllable manner. 


\section{CONCLUSIONS}

In summary, we have presented simple expressions for the RKKY interaction between magnetic impurities in strained nanotubes. We first validated our model by examining the role that the circumference of the nanotube plays in the decay rate as the nanotubes become more graphene-like, and found that ZZNT maintain their nanotube-like behavior for larger circumferences than ACNT. We have also shown that strain can change the magnitude, sign, and decay of these interactions in ACNTs. The amplification was particularly pronounced for center-adsorbed impurities where we showed that the symmetry breaking of the hexagonal lattice by uniaxial strain leads to a significantly slower decay rate between center-adsorbed impurities: $D^{-5}$ to $D^{-1}$ in armchair nanotubes. Zigzag nanotubes, meanwhile, display a wide range of amplification and switching effects with minor variations of the applied strain. These features are related to the transition from metallic to semiconducting behavior. Experiments to date searching for magnetism in disordered graphene seem to suggest paramagnetic, noninteracting moments [66]. Signatures of indirect exchange interactions between such moments in graphene are very difficult to detect due to their short- ranged nature, particularly if they adopt certain adsorption configurations. Amplification of these couplings using strain may provide a path to their detection in future experiments. The abrupt changes in interaction strength and behavior, demonstrated here for ZZNTs, suggest that strained nanotubes can act as switches for magnetoresistive behavior, which may find use in future spintronic devices. Thus strain presents itself as a way to control interactions between magnetic dopants in nanotubes, and the overall magnetic ordering of the material, in a reversible and controllable manner - an indispensable trait for future spintronic devices.

\section{ACKNOWLEDGMENTS}

The authors acknowledge financial support received from the Programme for Research in Third-Level Institutions PRTLI5 Ireland, the Irish Research Council for Science, Engineering, and Technology under the EMBARK initiative, and from Science Foundation Ireland under Grant No. SFI 11/RFP.1/MTR/3083. The Center for Nanostructured Graphene (CNG) is sponsored by the Danish National Research Foundation, Project No. DNRF58.
[1] N. Kheirabadi, A. Shafiekhani, and M. Fathipour, Superlattices Microstruct. 74, 123 (2014).

[2] G. Hrkac, J. Dean, and D. A. Allwood, Philos. Trans. R. Soc., A 369, 3214 (2011).

[3] P. Chen and G. Zhang, Sci. China: Phys., Mech. Astron. 56, 207 (2013).

[4] Y.-W. Son, M. L. Cohen, and S. G. Louie, Nature (London) 444, 347 (2006).

[5] W.-F. Tsai, C.-Y. Huang, T.-R. Chang, H. Lin, H.-T. Jeng, and A. Bansil, Nat. Commun. 4, 1500 (2013).

[6] I. Žutić, J. Fabian, and S. Das Sarma, Rev. Mod. Phys. 76, 323 (2004).

[7] A. Hirohata and K. Takanashi, J. Phys. D 47, 193001 (2014).

[8] The terms indirect exchange coupling and RKKY interaction are used synonymously throughout this paper.

[9] M. A. Ruderman and C. Kittel, Phys. Rev. 96, 99 (1954).

[10] T. Kasuya, Prog. Theor. Phys. 16, 45 (1956).

[11] K. Yosida, Phys. Rev. 106, 893 (1957).

[12] P. Bruno and C. Chappert, Phys. Rev. Lett. 67, 1602 (1991).

[13] P. Bruno and C. Chappert, Phys. Rev. B 46, 261 (1992).

[14] S. R. Power and M. S. Ferreira, Crystals 3, 49 (2013).

[15] M. A. H. Vozmediano, M. P. López-Sancho, T. Stauber, and F. Guinea, Phys. Rev. B 72, 155121 (2005).

[16] V. V. Cheianov and V. I. Fal'ko, Phys. Rev. Lett. 97, 226801 (2006).

[17] V. K. Dugaev, V. I. Litvinov, and J. Barnas, Phys. Rev. B 74, 224438 (2006).

[18] S. Saremi, Phys. Rev. B 76, 184430 (2007).

[19] L. Brey, H. A. Fertig, and S. Das Sarma, Phys. Rev. Lett. 99, 116802 (2007).

[20] E. H. Hwang and S. Das Sarma, Phys. Rev. Lett. 101, 156802 (2008).

[21] J. E. Bunder and H.-H. Lin, Phys. Rev. B 80, 153414 (2009).
[22] P. Venezuela, R. B. Muniz, A. T. Costa, D. M. Edwards, S. R. Power, and M. S. Ferreira, Phys. Rev. B 80, 241413 (2009).

[23] A. M. Black-Schaffer, Phys. Rev. B 81, 205416 (2010).

[24] B. Uchoa, T. G. Rappoport, and A. H. Castro Neto, Phys. Rev. Lett. 106, 016801 (2011).

[25] A. M. Black-Schaffer, Phys. Rev. B 82, 073409 (2010).

[26] S. R. Power and M. S. Ferreira, Phys. Rev. B 83, 155432 (2011).

[27] E. Kogan, Phys. Rev. B 84, 115119 (2011).

[28] H. Lee, J. Kim, E. R. Mucciolo, G. Bouzerar, and S. Kettemann, Phys. Rev. B 85, 075420 (2012).

[29] K. Szałowski, Phys. Rev. B 84, 205409 (2011).

[30] S. R. Power, F. S. M. Guimarães, A. T. Costa, R. B. Muniz, and M. S. Ferreira, Phys. Rev. B 85, 195411 (2012).

[31] F. Peng and W. Hongbin, Physica B (Amsterdam) 407, 3434 (2012).

[32] A. T. Costa, D. F. Kirwan, and M. S. Ferreira, Phys. Rev. B 72, 085402 (2005).

[33] D. F. Kirwan, C. G. Rocha, A. T. Costa, and M. S. Ferreira, Phys. Rev. B 77, 085432 (2008).

[34] J. Klinovaja and D. Loss, Phys. Rev. B 87, 045422 (2013).

[35] V. B. Shenoy, Phys. Rev. B 71, 125431 (2005).

[36] J. E. Bunder and J. M. Hill, J. Chem. Phys. 136, 154504 (2012).

[37] P. D. Gorman, J. M. Duffy, S. R. Power, and M. S. Ferreira, Phys. Rev. B 90, 125411 (2014).

[38] S. R. Power, P. D. Gorman, J. M. Duffy, and M. S. Ferreira, Phys. Rev. B 86, 195423 (2012).

[39] M. Sherafati and S. Satpathy, Phys. Rev. B 83, 165425 (2011).

[40] P. D. Gorman, J. M. Duffy, M. S. Ferreira, and S. R. Power, Phys. Rev. B 88, 085405 (2013).

[41] M. Sherafati and S. Satpathy, Phys. Rev. B 84, 125416 (2011). 
[42] J. M. Duffy, P. D. Gorman, S. R. Power, and M. S. Ferreira, J. Phys.: Condens. Matter 26, 055007 (2014).

[43] S. Latil, S. Roche, D. Mayou, and J.-C. Charlier, Phys. Rev. Lett. 92, 256805 (2004).

[44] S. Azevedo, C. Chesman, and J. R. Kaschny, Eur. Phys. J. B 74, 123 (2010).

[45] K. Nakada and A. Ishii, DFT Calculation for Adatom Adsorption on Graphene, Graphene Simulation, edited by J. Gong (InTech, Rijeka, Croatia, 2011), Chap. 1.

[46] W. M. C. Foulkes and R. Haydock, Phys. Rev. B 39, 12520 (1989).

[47] T. Ando, NPG Asia Materials 1, 17 (2009).

[48] J. N. Coleman, U. Khan, W. J. Blau, and Y. K. Gunko, Carbon 44, 1624 (2006).

[49] Q. Zhang, J.-Q. Huang, W.-Z. Qian, Y.-Y. Zhang, and F. Wei, Small 9, 1237 (2013).

[50] W. S. Su, J. Appl. Phys. 113, 244308 (2013).

[51] Y. R. Chen, C. I. Weng, and S. J. Sun, J. Appl. Phys. 104, 114310 (2008).

[52] L. Yang and J. Han, Phys. Rev. Lett. 85, 154 (2000).

[53] S. Sreekala, X.-H. Peng, P. M. Ajayan, and S. K. Nayak, Phys. Rev. B 77, 155434 (2008).

[54] A. Pullen, G. L. Zhao, D. Bagayoko, and L. Yang, Phys. Rev. B 71, 205410 (2005).
[55] J. W. Ding, X. H. Yan, J. X. Cao, D. L. Wang, Y. Tang, and Q. B. Yang, J. Phys.: Condens. Matter 15, L439 (2003).

[56] Y. He, C. Zhang, C. Cao, and H. P. Cheng, Phys. Rev. B 75, 235429 (2007).

[57] M. A. Cullinan and M. L. Culpepper, Phys. Rev. B 82, 115428 (2010).

[58] Y. Yoon and J. Guo, IEEE Trans. Electron Devices 54, 1280 (2007).

[59] E. D. Minot, Y. Yaish, V. Sazonova, J. Y. Park, M. Brink, and P. L. McEuen, Phys. Rev. Lett. 90, 156401 (2003).

[60] H. Fang, R. Z. Wang, M. Yan, S. Y. Chen, and B. Wang, Phys. Lett. A 375, 1200 (2011).

[61] A. Sharma, V. N. Kotov, and A. H. Castro Neto, Phys. Rev. B 87, 155431 (2013).

[62] J. Viana-Gomes, V. M. Pereira, and N. M. R. Peres, Phys. Rev. B 80, 245436 (2009).

[63] D. Midtvedt and A. Croy, arXiv:1504.06956.

[64] V. M. Pereira, A. H. Castro Neto, and N. M. R. Peres, Phys. Rev. B 80, 045401 (2009).

[65] V. M. Pereira and A. H. Castro Neto, Phys. Rev. Lett. 103, 046801 (2009).

[66] M. Sepioni, R. R. Nair, S. Rablen, J. Narayanan, F. Tuna, R. Winpenny, A. K. Geim, and I. V. Grigorieva, Phys. Rev. Lett. 105, 207205 (2010). 